\title{
Parámetros bioquímicos y hematológicos en ovinos de pelo con y sin sombra bajo condiciones desérticas
}

\section{Biochemical and hematological parameters in hair sheep with and without shade under desert conditions}

\author{
Arnulfo Vicente-Pérez ${ }^{1}$, Leonel Avendaño-Reyes ${ }^{2}$, Rubén Barajas-Cruz ${ }^{1}$, Ulises Macías-Cruz ${ }^{2}$, Abelardo \\ Correa-Calderón ${ }^{2}$, Ricardo Vicente-Pérez ${ }^{2}$, José Luis Corrales-Navarro ${ }^{2}$, Juan Eulogio Guerra-Liera ${ }^{3 *}$ \\ ${ }^{1}$ Facultad de Medicina Veterinaria y Zootecnia, Universidad Autónoma de Sinaloa. Boulevard San Ángel s/n, Fraccionamiento \\ San Benito, Predio Las Coloradas, Culiacán Sinaloa, México. \\ ${ }^{2}$ Instituto de Ciencias Agrícolas, Universidad Autónoma de Baja California. Ejido Nuevo León, Carretera a Delta s/n, CP. \\ 21705, Mexicali, Baja California, México. \\ ${ }^{3}$ Facultad de Agronomía, Universidad Autónoma de Sinaloa. Km. 17.5 Carretera Culiacán - El Dorado, CP. 80000. Culiacán, \\ Sinaloa, México. \\ *Autor de correspondencia: juaneulogioguerraliera @hotmail.com
}

Artículo científico recibido: 05 de julio de 2017 aceptado: 18 de febrero de 2018

RESUMEN. El objetivo del estudio fue determinar el efecto del uso de sombra en corderas de pelo bajo condiciones de estrés calórico sobre parámetros bioquímicos y hematológicos. Veinte corderas de pelo de cruza Dorper x Pelibuey fueron asignadas de forma aleatoria a uno de dos tratamientos: 1) corderas en corraletas individuales cubiertas con sombra (CS, $n=10)$; y 2) corderas en corraletas individuales sin acceso a sombra ( $S S, n=10)$. Se tomaron muestras de sangre por punción de la vena yugular, la colecta se realizó durante la mañana $(6: 00 \mathrm{~h})$, cada tercer día durante los $16 \mathrm{~d}$ del estudio. Los parámetros hematológicos se determinaron en muestras de sangre fresca, mientras que los metabolitos y electrolitos se determinaron en muestras de suero. Los datos se analizaron con un diseño de bloques al azar. Las corderas CS presentaron menor $(p<0.05)$ concentración de colesterol y triglicéridos que las corderas SS. Las concentraciones de $\mathrm{K}$ y Cl fueron mayores $(p<0.05)$ para el grupo SS en relación con el grupo CS. Las corderas CS presentaron mayor $(p<0.05)$ concentración de plaquetocrito, volumen corpuscular medio, hemoglobina corpuscular media y ancho de distribución eritrocitaria, pero menor concentración de hemoglobina en relación a corderas SS. Los resultados muestran que los ovinos de pelo tienen capacidad de adaptación a altas temperaturas, ya que dado el tiempo de exposición al calor, no se observaron cambios importantes en los componentes hematológicos y bioquímicos.

Palabras clave: Electrolitos, estrés calórico, hemoglobina, metabolitos, ovinos

ABSTRACT. The aim of this study was to determine the effect of shade use on the biochemical and hematological parameters in hair ewe lambs kept under conditions of caloric stress. Twenty Dorper $x$ Pelibuey cross hair ewe lambs were randomly assigned to one of two treatments: 1 ) ewe lambs in individual shaded pens (WS, $n=10)$; and 2) ewe lambs in individual pens without access to shade (NS, $n=10)$. Blood samples were taken by puncturing the jugular vein. The collection was made during the morning $(6: 00 \mathrm{~h})$, every third day during the $16-\mathrm{d}$ study. The hematological parameters were determined in fresh blood samples, while the metabolites and electrolytes were determined in serum samples. The data were analyzed with a randomized block design. WS ewe lambs had a lower $(p<0.05)$ concentration of cholesterol and triglycerides than the NS ewe lambs. The $\mathrm{K}$ and $\mathrm{Cl}$ concentrations were higher $(p<0.05)$ for the NS group than the WS group. WS ewe lambs showed a higher $(p<0.05)$ platelet concentration, mean corpuscular volume, mean corpuscular hemoglobin and erythrocyte distribution width, but lower hemoglobin concentration in relation to NS ewe lambs. The results show that hair sheep have the ability to adapt to high temperatures, since no significant changes were observed in their hematological and biochemical components as a result of their exposure time to heat. Key words: Electrolytes, caloric stress, hemoglobin, metabolites, sheep 


\section{INTRODUCCIÓN}

La producción de carne y lana de ovinos es la principal actividad económica de muchos países del mundo (McManus et al. 2010). El cambio climático es un fenómeno global que por sus efectos representa una amenaza para la viabilidad y sustentabilidad de la producción ovina en estos territorios (Gaughan et al. 2009), específicamente en las zonas ubicadas en el trópico (McManus et al. 2009), subtropico y áridas (Ganaie et al. 2013). En estas regiones, las temperaturas se elevan durante el verano, ocasionando que el equilibrio homeostático de los animales se deprima causando estrés calórico, acompañado de cambios fisiológicos y conductuales que efectan de forma negativa su productividad. Bajo estrés calórico, los ovinos activan mecanismos para contrarrestar los efectos, como la disminución del consumo de materia seca, incrementan la frecuencia respiratoria y en el consumo de agua (Marai et al. 2007, Al-Haidary et al. 2012). Estos cambios fisiológicos traen como consecuencia la activación de respuestas biológicas en el sistema nervioso autónomo y en la respuesta inmune (Moberg y Mench 2000). Las variaciones de la cantidad de sangre circulantes en el organismo y la tasa a la cual ésta se evapora de la piel y del tracto respiratorio están relacionadas con el incremento del consumo de agua (Richards 1973). Estas modificaciones en la cantidad de sangre circulante en el organismo pueden resultar en alteraciones de parámetros hematológicos, bioquímicos y hormonales debido a que la sangre es un indicador importante del estrés calórico en animales (Sullivan et al. 2011, Kamal et al. 2016). Además, la sangre es altamente sensible a los cambios ambientales, por lo que cualquier cambio cuantitativo y morfológico en las células sanguíneas se vincula de forma directa con el estado fisiológico o patológico del animal (Bhan et al. 2012).

El análisis y cuantificación de metabolitos, electrolitos y componentes hematológicos proveen evidencias de la capacidad de adaptación de un animal a los cambios ambientales mediante la altera- ción de su actividad metabólica (Sejian et al. 2010a). Al respecto Valtorta y Gallardo (2004) reportan que la utilización de sombras en los corrales puede reducir la carga de calor en los animales. Pero hay pocos estudios sobre el efecto que tiene la utilización de sombras en los componentes sanguíneos de ovinos de pelo en regiones áridas de nuestro país. Por lo anterior, el objetivo del estudio fue conocer el efecto de la presencia de sombra sobre parámetros hematológicos y bioquímicos en ovinos de pelo bajo altas temperaturas.

\section{MATERIALES Y MÉTODOS}

\section{Sitio y duración del estudio}

El estudio se realizó en el verano de 2015 en la unidad experimental ovina del Instituto de Ciencias Agrícolas, de la Universidad Autónoma de Baja California (ICA-UABC), ubicada en el Valle de Mexicali, Baja California, México (32.8 LN, $114.6^{\circ}$ LO). Las condiciones climáticas predominantes en son desérticas, que se caracterizan por ser extremadamente secas y calientes, con temperatura máxima en verano de $46^{\circ} \mathrm{C}$ y mínima en el invierno de $-2{ }^{\circ} \mathrm{C}$; con precipitación promedio anual de 85 mm (García 1985).

\section{Animales experimentales y tratamientos}

El cuidado y manejo de los animales se realizó de acuerdo con la Norma Oficial Mexicana NOM051 ZOO-1995, que especifica el trato humanitario en la movilización y manejo de animales. El estudio también fue autorizado por el Comité de Ética de la UABC. Se utilizaron 20 corderas de pelo cruzas Dorper $x$ Pelibuey con peso promedio de $30.4 \pm 3.4 \mathrm{~kg}$ y edad de cuatro meses. Las corderas nacieron y fueron criadas en la posta experimental del ICA-UABC, las cuales se pesaron al inicio del experimento para utilizarlo como factor de bloqueo, asignando de forma aleatoria cada cordera a uno de los tratamientos: 1) ovejas en corraletas individuales cubiertas de sombra permanentemente ( CS = con sombra, $n=10$ ); y 2 ) ovejas en corraletas individuales $\sin$ acceso a sombra (SS $=\sin$ som- 
bra, $\mathrm{n}=10$ ), para tener 10 bloques. Las corraletas se ensamblaron con paneles de alambre con dimensiones de $1.0 \times 1.5 \mathrm{~m}$, con comederos y bebederos individuales en la parte frontal. En el tratamiento CS, el material del techo fue lámina galvanizada que se colocó a $2.5 \mathrm{~m}$ de altura, con inclinación para bloquear el $100 \%$ de la radiación. Adicionalmente, en ese mismo grupo, la parte lateral superior de la malla ciclónica se cubrió con malla-sombra para bloquear la radiación solar en un $30 \%$, para que los animales no reciban la luz del sol de forma directa.

\section{Alimentación y manejo de las corderas}

La dieta ofrecida tuvo $66 \%$ de grano molido de trigo, $12 \%$ de heno de alfalfa, $11.5 \%$ de paja de trigo, $8 \%$ de harina de soya, $1 \%$ de piedra caliza, $1 \%$ de fosfato dicálcico y $0.5 \%$ de sal común. La dieta aportó $12.98 \%$ de proteína cruda y 2.79 Mcal de $\mathrm{EM} \mathrm{kg}^{-1}$ de MS. Esta se proporcionó a las 7:00 y 17:00 $\mathrm{h}$ en ambos tratamientos. Dado que el consumo de alimento se midió todos los dias, la cantidad de alimento se ajustó de acuerdo a lo consumido el día previo, de forma que el rechazo fuera de aproximadamente el $5 \%$.

\section{Evaluación de variables}

Las variables climáticas, temperatura ambiental y humedad relativa se midieron con higrotermógrafos que se colocaron en la parte superior de los corrales experimentales, los cuales registraron estas variables cada $10 \mathrm{~min}$. Las variables se utilizaron para construir el índice temperaturahumedad (ITH). Que se calculó con la fórmula propuesta por Hahn (1999):

$$
I T H=0.81 * T e m p+\frac{H R}{100} *(T e m p-14.40)+46.4
$$

Donde: ITH es el índice temperatura-humedad, Temp es la temperatura ambiente y $\mathrm{HR}$ es la humedad relativa.

Para el análisis de metabolitos, electrolitos y componentes hematológicos se tomaron muestras de sangre en tubos vacutainer de 10 y $4 \mathrm{~mL}$ mediante la técnica de venopunción de la vena yugular. La colecta de sangre se realizó a las 6:00 h, cada tercer día durante el experimento. Para el análisis de componentes hematológicos se utilizaron las muestras de sangre fresca obtenidas en tubos de 4 $\mathrm{mL}$ que contenían EDTA- $\mathrm{K}_{2}$, las cuales se transportaron al laboratorio para su análisis en un equipo automatizado Auto Hematology Analyzer (MINDRAY, BC-2800 Vet). Las muestras de sangre colectadas en tubos de $10 \mathrm{~mL}$ se centrifugaron a $3500 \mathrm{rpm}$ por $15 \mathrm{~min}$ a $10{ }^{\circ} \mathrm{C}$, para separar el suero por duplicado en viales de $2 \mathrm{ml}$ y almacenar a $-20{ }^{\circ} \mathrm{C}$ para el posterior análisis de glucosa, colesterol, urea, triglicéridos, proteína total y electrolitos ( $\mathrm{Na}, \mathrm{K}$ y $\mathrm{Cl}$ ). Los metabolitos se determinaron con un equipo de química sanguínea (Model DT-60, Johnson Co.), mientras que los electrolitos se determinaron en un Electrolyte Analyzer LW E60A.

\section{Análisis estadísticos}

Todas las variables se sometieron a un análisis de varianza bajo un diseño de bloques completos al azar con mediciones repetidas en el tiempo, usando el procedimiento GLM del SAS (2004), donde la medición repetida correspondió a los días de muestreo ( $5 \mathrm{~d})$. Las medias fueron separadas con el comando PDIFF a un nivel de significancia de 5\%.

\section{RESULTADOS}

\section{Variables climáticas}

La Tabla 1 muestra las variables climáticas que se registraron durante el experimento. Como se puede observar, para ambos tratamientos se muestran las temperaturas máximas, mínimas y promedios, humedad relativa e ITH. Para el tratamiento con sombra, la temperatura máxima alcanzó los 45 ${ }^{\circ} \mathrm{C}$, con promedio de $34.1{ }^{\circ} \mathrm{C}$, mientras que en el tratamiento sin sombra la temperatura máxima fue de $50{ }^{\circ} \mathrm{C}$ con promedio de $35.3{ }^{\circ} \mathrm{C}$. Los valores máximos, mínimos y promedios de humedad relativa fueron iguales para ambos tratamientos. Pero la ITH fue ligeramente mayor para el tratamiento sin sombra en comparación con el tratamiento con sombra, con promedios de 82.2 y 81.2 unidades res- 
pectivamente, observándose condiciones climáticas adversas en ambos tratamientos.

Tabla 1. Variables climáticas registradas durante el estudio en los tratamientos con $y$ sin sombra durante el verano.

\begin{tabular}{lcc}
\hline Clima & \multicolumn{2}{c}{ Tratamiento } \\
& Con sombra & Sin sombra \\
\hline Promedio & 34.1 & 35.3 \\
Máxima & 45 & 50 \\
Mínima & 20.4 & 18.6 \\
& Humedad & Relativa $(\%)$ \\
Promedio & 42.7 & 42.7 \\
Máxima & 93.4 & 93.4 \\
Mínima & 8.7 & 8.7 \\
& ITH & (unidades) \\
Promedio & 81.2 & 82.2 \\
Máximo & 88.4 & 92.8 \\
Mínimo & 62.7 & 65.3 \\
\hline
\end{tabular}

\section{Metabolitos sanguíneos}

Los metabolitos sanguíneos bajo el efecto de la sombra y sin sombra se presentan en la Tabla 2. Se observa efecto de tratamiento en las concentraciones de colesterol y triglicéridos. En las corderas con sombra se tuvo la menor concentración de colesterol y triglicéridos $(p<0.05)$, con respecto a las corderas sin sombra. Mientras que los metabolitos no fueron afectados por efecto del tratamiento; también se observa que el consumo de alimento fue similar en ambos tratamientos, en tanto que los animales sin sombra consumieron $15 \%$ más agua que los animales con sombra $(p<0.05)$.

\section{Electrolitos sanguíneos}

El contenido de electrolitos sanguíneos bajo el efecto de los tratamientos se presenta en la Tabla 3. Las concentraciones de potasio y cloro fueron mayores $(p<0.05)$ en el tratamiento sin sombra con respecto al tratamiento con sombra (5.23 vs $4.79 \mathrm{mmol}$ ) (113.14 vs $112.05 \mathrm{mmol}$ ). Mientras que las concentraciones de sodio no fueron afectadas por el tratamiento.

\section{Componentes hematológicos}

En la Tabla 4, se observa que las corderas que se encontraban con sombra presentaron mayores $(p$
$<0.05)$ concentraciones de volumen corpuscular medio (VCM), hemoglobina corpuscular media (HCM), ancho de distribución eritrocitaria (ADE), amplitud de distribución del tamaño plaquetario (ADTP) y plaquetocrito (PCT), pero menor concentración de hemoglobina en relación con el tratamiento sin sombra. Mientras que el resto de los componentes hematológicos no fueron afectados por el tratamiento.

\section{DISCUSIÓN}

\section{Condiciones climáticas}

Las condiciones climáticas durante el experimento fueron estresantes para las corderas en ambos tratamientos, debido a que los promedios de temperatura diaria excedieron el límite superior de la zona de confort indicado para ovinos de 24 a $27{ }^{\circ} \mathrm{C}$ (Fuquay 1981). Adicionalmente, el estrés calórico fue severo debido a que la ITH tuvo promedios superiores de 80 unidades en ambos tratamientos (Marai et al. 2007, 2008). El cual fue ligeramente mayor para el tratamiento sin sombra en comparación con el tratamiento con sombra. Los valores máximos, mínimos y promedio de humedad relativa fueron iguales en ambos tratamientos. Mientras que la temperatura, HR e ITH confirman que las corderas tuvieron condiciones estresantes durante el experimento.

\section{Metabolitos sanguíneos}

En la época de verano, las condiciones ambientales afectan de forma negativa a los animales, por lo que la cuantificación de metabolitos en sangre es una forma para tener evidencias de la capacidad de adaptación a los cambios ambientales (Sejian et al. 2010a). El efecto del estrés calórico en el estado metabólico de ovinos ha sido poco estudiado, con resultados contrastantes, debido a que esta forma de estrés depende de la intensidad y duración de los factores de estrés, la especie y las características genéticas del animal (Sejian et al. 2010b, Alhidary et al. 2012, Indu et al. 2015). 
Vicente-Pérez et al.

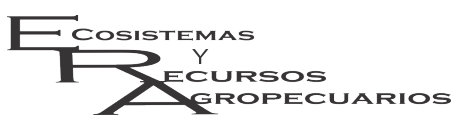

Bioquímica sanguínea y hematología en ovinos

Ecosist. Recur. Agropec.

5(14):259-269,2018

Tabla 2. Promedios y errores estándar de consumo de agua y alimento, así como de metabolitos sanguíneos en corderas con y sin sombra durante el estudio.

\begin{tabular}{lccc}
\hline Variable & \multicolumn{3}{c}{ Tratamientos } \\
& Con Sombra & Sin Sombra & $\mathrm{EE}$ \\
\hline Consumo alimento $\left(\mathrm{g} \mathrm{d}^{-1}\right)$ & $1089.9^{a}$ & $974.0^{a}$ & 62.92 \\
Consumo agua $\left(\mathrm{L} \mathrm{d}^{-1}\right)$ & $3.77^{a}$ & $4.49^{b}$ & 0.21 \\
Colesterol $\left(\mathrm{mg} \mathrm{dL}^{-1}\right)$ & $49.37^{a}$ & $61.39^{b}$ & 2.24 \\
Triglicéridos $\left(\mathrm{mg} \mathrm{dL}^{-1}\right)$ & $44.78^{a}$ & $50.20^{b}$ & 2.57 \\
Glucosa $\left.(\mathrm{mg} \mathrm{dL})^{-1}\right)$ & $77.04^{a}$ & $82.78^{b}$ & 2.46 \\
Proteína total $\left(\mathrm{mg} \mathrm{dL}^{-1}\right)$ & $7.01^{a}$ & $6.98^{a}$ & 0.06 \\
Urea (mg dL & $39.80^{a}$ & $35.45^{a}$ & 1.13 \\
\hline ab Promedios con distinta literal en hilera difieren $(p<0.05), \mathrm{EE}:$ \\
error estándar.
\end{tabular}

Tabla 3. Promedios y errores estándar de electrolitos sanguíneos en corderas con y sin sombra durante el estudio.

\begin{tabular}{cccc}
\hline Variable & \multicolumn{3}{c}{ Tratamientos } \\
& Con Sombra & Sin Sombra & $\mathrm{EE}$ \\
\hline $\mathrm{K}(\mathrm{mmol})$ & $4.79^{a}$ & $5.23^{b}$ & 0.10 \\
$\mathrm{Na}(\mathrm{mmol})$ & $141.74^{a}$ & $142.21^{a}$ & 1.96 \\
$\mathrm{Cl}(\mathrm{mmol})$ & $112.05^{a}$ & $113.14^{b}$ & 0.30 \\
\hline$a b$ Promedios con distinta literal en hilera difieren \\
$(p<0.05), \mathrm{K}$ : Potasio, Na: Sodio, Cl: Cloro, EE: \\
Error estándar.
\end{tabular}

Tabla 4. Promedios y errores estándar de variables hematológicas en corderas con sombra y sin sombra.

\begin{tabular}{lccc}
\hline Variables & \multicolumn{3}{c}{ Tratamientos } \\
& Con Sombra & Sin Sombra & EE \\
\hline GR (X 10 $\left.0^{12} \mathrm{~L}\right)$ & $13.14^{a}$ & $13.72^{a}$ & 0.31 \\
Hemoglobina (g dl-1) & $12.16^{a}$ & $12.41^{b}$ & 0.24 \\
Hematocrito (\%) & $40.20^{a}$ & $40.54^{a}$ & 0.73 \\
VCM (X 10 $\left.{ }^{15} \mathrm{~L}\right)$ & $31.27^{a}$ & $29.70^{b}$ & 0.45 \\
HCM (Pg) & $9.37^{a}$ & $9.00^{b}$ & 0.02 \\
CCMH (g dl $\left.{ }^{-1}\right)$ & $30.05^{a}$ & $30.54^{a}$ & 0.21 \\
ADE (\%) & $18.04^{a}$ & $17.29^{b}$ & 0.18 \\
Plaquetas, X $10^{9} \mathrm{~L}$ & $601.5^{a}$ & $555.5^{a}$ & 25.5 \\
Vol. Pqts, X $10^{15} \mathrm{~L}$ & $3.96^{a}$ & $3.57^{a}$ & 0.18 \\
ADTP & $15.32^{a}$ & $15.15^{b}$ & 0.04 \\
PCT & $0.22^{a}$ & $0.19^{b}$ & 0.009 \\
\hline
\end{tabular}

$\overline{a b}$ Promedios con distinta literal en hilera difieren $(p<0.05)$, GR: Glóbulos rojos, VCM: Volumen corpuscular medio, HCM: hemoglobina corpuscular media, CCMH: concentración corpuscular media de hemoglobina, ADE: ancho de distribución de eritrocitos, ADTP: ancho de distribución del tamaño plaquetario, Vol. Pqts: Volumen de plaquetas, EE: Error estándar.

El mayor contenido de colesterol en el tratamiento sin sombra coincide con Liu et al. (2012), quienes indican que el estrés experimentado en las corderas de este tratamiento incrementó la glicogenólisis, por aumento en las catecolaminas y glucocorticoides. Lo que fue confirmado por Caroprese et al. (2010), quienes reportan menor concentración de glucosa en ovinos con sombra que los expuestos a altas temperaturas. Por otro lado, el nivel de glicemia experimentado por los rumiantes podría contribuir a la valoración del estatus energético, pero considerando el nivel de insulina y de los ácidos grasos libres, los cuales están fuertemente asociados con el estatus nutricional, indica niveles altos en un balance de energía negativa y niveles bajos un balance de energético positivo (Caldeira et al. 2007). Mientras que Alhidary et al. (2012) reportan que ovinos sometidos a condiciones de estrés calórico por siete días presentaron niveles sanguíneos de glucosa, PT, colesterol, triglicéridos y urea sin cambios importantes. Pero la concentración de glucosa y colesterol son los metabolitos propensos a alteraciones en climas cálidos (Ribeiro et al. 2014). Al respecto Sejian et al. (2010b) e Indu et al. (2015) reportan que en condiciones de estrés calórico, los niveles de glucosa y colesterol disminuyen. Lo que puede deberse a la hemodilución, debido al incremento del consumo de agua y disminución del consumo de alimento (Marai et al. 2008, Sejian et al. 2010a), o por mayor utilización de glucosa para producir la energía que requiere la actividad muscular del sistema respiratorio debido al incremento de la frecuencia respiratoria (Srikandakumar et al. 2003, Sejian et al. 2010b). Como consecuencia de la caída en los niveles plasmáticos de glucosa, se tiene una disminución del colesterol por el incremento de la utilización de ácidos grasos, 
como respuesta a la demanda de energía (Rasooli et al. 2004); además, la disminución de los niveles de triglicéridos en ovinos bajo condiciones cálidas se puede deber al incremento en la lipólisis, como resultado de la reducción de los niveles plasmáticos de glucosa (Indu et al. 2015). Otro de los metabolitos sanguíneos afectado por la exposición a las altas temperaturas es el colesterol (Marai et al. 2008), se ha observado que su concentración disminuye con el incremento de la temperatura (Marai et al. 2007). Este cambio puede deberse a la dilución del mismo por el incremento de agua en el cuerpo; o también por una baja en la concentración de acetato, que es el principal precursor en la síntesis de colesterol (Marai et al. 2008). El incremento de los niveles de la hormona glucocorticoide en animales estresados por calor, puede ser otro factor causante de la disminución del colesterol en sangre (Marai et al. 2008). Por lo que se esperaba deshidratación de los animales como respuesta al estrés por calor, y que el contenido de glucosa y colesterol fueran afectados de forma negativa por efecto del mayor consumo de agua de los animales sin sombra, pero los niveles de ambos tratamientos se encontraron dentro del rango normal de referencia (Kaneko et al. 1997, Wang et al. 2015).

\section{Electrolitos sanguíneos}

Dentro de los ajustes fisiológicos observados en ovejas expuestas a ambientes cálidos se incluye el incremento en la frecuencia respiratoria y el consumo de agua, así como reducción en el consumo de materia seca (Nardone et al. 2010), que actúan como mecanismos para disminuir el calor corporal acumulado. Pero las mismas condiciones de calor incrementan las pérdidas de agua, lo que conduce a deshidratación. Por otra parte, estos ajustes fisiológicos alteran las condiciones ácido-básicas de la sangre por pérdida de $\mathrm{CO}_{2}$, reduciendo la concentración de ácido carbónico $\left(\mathrm{H}_{2} \mathrm{CO}_{3}\right)$ e incrementando la concentración de bicarbonato $\left(\mathrm{HCO}_{3}-\right)$ como consecuencia de la disminución de las concentraciones de $\mathrm{Cl}$ - (Olanrewaju et al. 2007); pero este efecto puede ser revertido por la naturaleza alcalogénica de $\mathrm{Na}$ y $\mathrm{K}$. Por lo que las condiciones de estrés calórico desafían la capacidad del animal para mantener un equilibrio mineral y homeostático (Sivakumar et al. 2010).

En ovejas Dorper x Pelibuey, Macías-Cruz et al. (2015) observaron que en la época de verano los niveles de $\mathrm{Cl}$ disminuyen, pero se incrementa el $\mathrm{Na}$ en comparación con la época de primavera. Por otro lado, Al-Haidary et al. (2012) en ovejas Najdi reportan mayores concentraciones de $\mathrm{Na}$ y $\mathrm{Cl}$ en verano con respecto al invierno. Mientras que Rashid et al. (2013), trabajando con ovejas indígenas nativas no encontraron efecto importante de la época en el contenido de electrolitos; en tanto que Fadare et al. (2013) observaron que ovinos West África disminuyeron sus niveles plasmáticos de $\mathrm{Na}$ y $\mathrm{K}$ durante el verano. En otras especies animales también se han reportado resultados contradictorios con respecto a los niveles de electrolitos sanguíneos en diferentes epocas del año. Al respecto Srikandakumar et al. (2003) y Singh et al. (2012) señalan que las concentraciones de $\mathrm{Na}, \mathrm{K}$ y $\mathrm{Cl}$ disminuyen en condiciones de estrés calórico por $16 \mathrm{~d}$ en comparación con animales en ambientes termoneutrales. Resultados similares fueron reportados por Hooda y Upadhyay (2014), quienes observaron que los niveles de electrolitos en cabras se redujeron bajo condiciones de estrés calórico. A pesar de que los estudios anteriores fueron bajo condiciones de clima cálido, los niveles de electrolitos fueron divergentes, lo que puede atribuirse a las diferencias en el tiempo de exposición al calor, la alimentación, la especie animal e incluso la raza, entre otros factores. En condiciones cálidas, el consumo de agua es el principal determinante de la ingesta de alimento en los rumiantes, aúnque las mismas condiciones de calor incrementan las pérdidas de agua, deshidratación y pérdida de electrolitos; lo que puede ser un factor importante en el descenso de electrolitos.

Los niveles de $\mathrm{K}$ y $\mathrm{Cl}$ fueron mayores en las corderas en el tratamiento sin sombra que en el con sombra. Lo que se atribuye a que los animales sin sombra consumieron más agua que los animales con sombra. Con niveles de electrolitos sanguíneos en ambos tratamientos dentro del rango de referencia reportado por Kaneko et al. (1997), lo cual 
puede indicar que el tiempo de exposición al calor no fue suficientemente para provocar cambios en los electrolitos sanguíneos, evidenciando respuestas homeostáticas en el animal sobre los factores de estrés ambiental. Al respecto Silanikove (2000) menciona que los animales pueden activar mecanismos hipotalámicos de control del agua, mediante la estimulación y secreción de la hormona vasopresina, evitando la excreción de agua y pérdida de iones de electrolitos.

El mecanismo de termorregulación más eficiente que expresan los ovinos para disipar el calor acumulado bajo condiciones de estrés térmico, es el incremento en la frecuencia respiratoria. Los ovinos bajo estas condiciones ambientales presentan mayor frecuencia respiratoria (Barnes et al. 2004). La utilización de sombras en los corrales permite disminuir entre 30 y $50 \%$ la carga calórica total, mejorando el estatus fisiológico y de confort (Collier et al. 2006). Por lo que, utilizando corrales con sombra se disminuye la frecuencia respiratoria en los animales, mejora el estatus acido-básico y electrolítico. Al respecto Aengwanich et al. (2011), López et al. (2014) y Barragán-Hernández et al. (2015) reportan que para ovinos y bovinos el uso de sombra en corrales disminuye la frecuencia respiratoria. Pero son pocos los estudios que indican que la sombra en los rumiantes tiene efecto en los niveles de electrolitos. Mientras que Okoruwa (2014) encontraron que las cabras en corrales provistos de sombra presentaron menor concentración de $\mathrm{Na}$ y $\mathrm{K}$ al compararla con cabras sin sombra. Al respecto Barragán-Hernández et al. (2015) encontraron que, en bovinos bajo pastoreo, los niveles de $\mathrm{Na}, \mathrm{K}, \mathrm{y}$ $\mathrm{Cl}$ fueron mayores que los que tuvieron animales que pastaban en áreas con árboles. Otro aspecto que pudo influir en los resultados, fue que los animales sin acceso a sombra lograron recuperarse por encontrarse en corrales sin techo y expuestas a las corrientes directas del viento, por lo que pudieron disipar el calor.

\section{Componentes hematológicos}

Bajo condiciones de clima cálido, las concentraciones de los parámetros hematológicos en animales se modifican. La mayor concentración $\mathrm{de} \mathrm{Hb}$ en el tratamiento sin sombra puede estar asociado a mecanismo de adaptación de las corderas para proporcionar la cantidad necesaria de agua hacia el sistema circulatorio, para enfriarse por evaporación (Kamal et al. 2016). Además, las corderas en este tratamiento evidenciaron que hubo condiciones de estrés por el incremento en los niveles de $\mathrm{Hb}$ en la sangre, como consecuencia de mayor demanda de oxígeno, para activar los mecanismos fisiológicos de termorregulación (Ribeiro et al. 2014, Al-Dawood 2017). Además de estar relacionados con el incremento de la actividad metabólica para satisfacer las demandas energéticas de mantenimiento y crecimiento. A pesar de ello, el promedio de $\mathrm{Hb}$ detectado en corderas con y sin acceso a sombra se encuentra dentro del rango normal de referencia de 9 a $16 \mathrm{~g} \mathrm{dL}^{-1}$ (Wang et al. 2015, Ahmadi-Hamedani et al. 2016). Los resultados obtenidos coinciden con lo señalado por Silva et al. (2011), quienes reportan valores de $\mathrm{Hb}$ mayores en animales sin sombra que con acceso a sombra. Estos resultados se pueden deber a las condiciones de estrés calórico que se tuvo por hemoconcentración por la pérdida de agua por medios evaporativos. Al respecto, Okoruwa (2014) encontró que las cabras que permanecieron mayor tiempo en condiciones de sol presentaron mayor concentración de hemoglobina en comparación con cabras que tuvieron mayor tiempo en sombra. Resultados que pueden atribuirse a la mayor acumulación de calor corporal en los animales, por lo que incrementaron los niveles de hemoglobina para poder cubrir las necesidades de oxigenación en la pérdida de calor. Al respecto Aengwanich et al. (2011) reportan que no encontraron diferencias significativas en los niveles de hemoglobina en novillas con y sin acceso a sombra. Mientras que Wojtas et al. (2014) y Srikandakumar et al. (2003), reportan que en ovinos bajo estrés calórico disminuyeron los niveles de hemoglobina.

La menor concentración de VCM, HCM, ADE, ADTP y PCT en corderas sin sombra esta asociado a diferentes fenómenos. Al respecto Rowlands et al. (1977) afirman que el descenso de los niveles 
de estos componentes se debe a la hemodilución en la sangre por el incremento del consumo de agua. Mientras que Coopo et al. (2002) afirman que la disminución de estos valores se asocia con la reducción de aportes nutricionales de los pastizales en la época de verano. En tanto que Larson et al. (1980) lo relacionan con la necesidad fisiológica de reducir el calentamiento metabólico mediante la reducción del requerimiento de oxígeno celular para compensar el calor ambiental. A pesar de que las corderas sin sombra presentaron menores concentraciones de VCM, HCM, ADE, ADTP y PCT, todas las concentraciones se encontraron en el límite inferior del rango de referencia en ambos tratamientos (Kaneko et al.1997, Wang et al. 2015, AhmadiHamedani et al. 2016).

\section{CONCLUSIONES}

Las variables hematológicas, electrolitos y metabolitos medidos en corderas con y sin acceso a sombra no tuvieron diferencias importantes, pero se sabe que bajo condiciones de estrés calórico severo los animales sufren cambios en sus procesos fisiológicos, bioquímicos y hematológicos, al respecto se ha reportado que con la utilización de sombras en los corrales se les proporciona alivio y confort. Se recomienda utilizar periodos de estrés calórico crónico para observar resultados más consistentes en los perfiles hematológicos y de bioquímica sanguínea.

\section{AGRADECIMIENTOS}

Al Cuerpo Académico Fisiología y Genética Animal del ICA-UABC, al proyecto de investigación No. 2229 de la 18a Convocatoria Interna de Apoyo a Proyectos de Investigación de la Coordinación de Posgrado e Investigación de la Universidad Autónoma de Baja California.

\section{LITERATURA CITADA}

Aengwanich W, Kongbuntad W, Boonsorn T (2011) Effects of shade on physiological changes, oxidative stress, and total antioxidant power in Thai Brahman cattle. International Journal of Biometeorology 55: $741-748$.

Ahmadi-Hamedani M, Ghazvinian K, Atyabi N, Khanalizadeh P, Masoum MA, Ghodrati MS (2016) Hematological reference values of healthy adult Sangsari sheep (Iranian fat-tailed sheep) estimated by Reference Value Advisor Comp. Journal of Clinical Pathology 25: 459-464.

Al-Dawood A (2017) Towards heat stress management in small ruminants - a review. Annals of Animal Science 17: 59-88.

Al-Haidary AA, Aljumaah RS, Alshaikh MA, Abdoun KA, Samara EM, Okab AB, et al. (2012) Thermoregulatory and physiological responses of Najdi sheep to environmental heat load prevailingin. Saudi Arabia Pakistan Veterinary Journal 2: 515-519.

Alhidary IA, Shini S, RAM ALJ, Gaughan JB (2012) Physiological responses of Australian Merino wethers exposed to high heat load. Journal of Animal Science 90: 212-220.

Barnes A, Beatty D, Taylor E, Stockman C, Maloney S, McCarthy M (2004) Physiology of heat stress in cattle and sheep. Meat and Livestock Australia 209: 1-36.

Barragán-Hernández WA, Mahecha-Ledesma L, Cajas-Girón YS (2015) Variables fisiológicas-metabólicas de estrés calórico en vacas bajo silvopastoreo y pradera sin árboles. Agronomía Mesoamericana 26: 211-223.

Bhan C, Singh SV, Hooda OK, Upadhyay RC, Beenam, Vaidya M (2012) Influence of temperature variability on physiological, hematological and biochemical profile of growing and adult sahiwal cattle. Journal of Environmental Research And Development 7: 986-984. 
Caldeira RM, Belo AT, Santos CC, Vázquez MI, Portugal AV (2007) The effect of long-term feed restriction and over-nutrition on body condition score, blood metabolites and hormonal profiles in ewes. Small Ruminant Research. 68: 242-255.

Caroprese M, Albenzio M, Marzano A, Schena L, Annicchiarico G, Sevi A (2010) Relationship between cortisol response to stress and behavior, immune profile, and production performances of dairy ewes. Journal of Dairy Science 93: 2395-2403.

Collier RJ, Dahl GE, Van Baale MJ (2006) Major advances associated with environmental effects on dairy cattle. Journal of Dairy Science 89: 1244-1253.

Coopo NB, Coopo JA, Revidatti MA, Capellari A, Navamuel JM, Fioranelli SA (2002) Cambios del eritrograma en vaquillonas cruza cebú, suplementadas con pulpa de citrus. Revista Veterinaria 13: 1-2.

Fadare AO, Peters SO, Yakubu A, Sonibare AO, Adeleke MA, Ozoje MO, et al. (2013) Physiological and haematological indices suggest superior heat tolerance of white-coloured West African Dwarf sheep in the hot humid tropics. Tropical Animal Health and Production 45: 157-165.

Fuquay JW (1981) Heat stress as is affects animal production. Journal of Animal Science 52: 64-174.

Ganaie AH, Ghasura RS, Mir NA, Bumla NA, Sankar G, Wani SA (2013) Biochemical and physiological changes during thermal stress in bovines: A review. Iranian Journal of Applied Animal Science 3: 423-430.

García E (1985) Modificaciones al sistema de clasificación climática de Koeppen. 3ra. Edición Universidad Nacional Autónoma de México. México. 90p.

Gaughan JB, Mader TL, Holt SM, Sullivan ML, Hahn GL (2009) Assessing the heat tolerance of 17 beef cattle genotypes. International Journal of Biometeorology 54: 617-627.

Hooda OK, Upadhyay RC (2014) Physiological responses, growth rate and blood metabolites under feed restriction and thermal exposure in kids. Journal of Stress Physiology and Biochemistry 10: 214-227.

Indu S, Sejian V, Naqvi SMK (2015) Impact of simulated heat stress on growth, physiological adaptability, blood metabolites and endocrine responses in Malpura ewes under semiarid tropical environment. Animal Production Science 55: 766-776.

Kamal R, Dutt T, Patel M, Dey A, Chandran PC, Bharti PK, et al. (2016) Behavioural, biochemical and hormonal responses of heat-stressed crossbred calves to different shade materials. Journal of Applied Animal Research 44: 347-354.

Kaneko JJ, Harvey JW, Bruss ML (1997) Clinical Biochemistry of Domestic Animals. Academic Press. 5th Edition. USA. 932p.

Larson LL, Mabruck HS, Lowry SR (1980) Relationship between early postpartum blood composition and reproductive performance in dairy cattle. Journal of Dairy Science 63: 283-289.

Liu HW, Cao Y, Zhou DW (2012) Effects of shade on welfare and meat quality of grazing sheep under high ambient temperature. Journal of Animal Science 90: 4764-4770.

López R, Pinto-Santini L, Perozo D, Pineda J, Oliveros I, Chacón T, et al. (2015) Thermal comfort and growth of West African lambs grazing with and without access to artificial shade. Archivos de Zootecnia 64: 139-146.

Macías-Cruz U, López-Baca MA, Vicente R, Mejía A, Álvarez FD, Correa-Calderón A, et al. (2015) Effects of seasonal ambient heat stress (spring vs. summer) on physiological and metabolic variables in hair sheep located in an arid region. International Journal of Biometeorology 60: 1279-1286. 
Marai IFM, El-Darawany AA, Fadiel A, Abdel-Hafez MAM (2008) Reproductive performance traits as affected by heat stress and its alleviation in sheep. Tropical and Subtropical Agroecosystems 8: 209-234.

Marai IFM, El-Darawany AA, Fadiel A, Abdel-Hafez MAM (2007) Physiological traits as affected by heat stress in sheep - a review. Small Ruminant Research 71: 1-12.

McManus C, Paludo GR, Louvandini H, Gugel R, Bastos SLC, Rezende SP (2009) Heat tolerance in Brazilian sheep: Physiological and blood parameters. Tropical Animal Health and Production 41: 95-101.

McManus C, Louvandini H, Gugel R, Bastos SLC, Bianchini E, Moreno BFE, et al. (2010) Skin and coat traits in sheep in Brazil and their relation with heat tolerance. Tropical Animal Health and Production 43: $121-126$.

Moberg GP (2000) Biological responses to stress. Implications for animal welfare. In: Moberg G.P. and Mench JP (eds) The biology of animal stress: Basic principles and implications for animal welfare. CAB International. Wallingford, UK. pp.1-41.

Nardone A, Ronchi B, Lacetera N, Ranieri MS, Bernabucci U (2010) Effects of climate changes on animal production and sustainability of livestock systems. Livestock Science 130: 57-69.

Okoruwa MI (2014) Effect of heat stress on thermoregulatory, live bodyweight and physiological responses of dwarf goats in southern Nigeria. European Journal of Scientific Research 10: 1857 - 7881.

Olanrewaju HA, Thaxton JP, Dozier WA, Branton SL (2007) Electrolyte diets, stress, and acid-base balance in broiler chickens. Poultry Science 86: 1363-1371.

Rashid MM, Hossain MM, Azad MAK, Hashem MA (2013) Long term cyclic heat stress influences physiological responses and blood characteristics in indigenous sheep. Bangladesh Journal of Animal Science 42: $96-100$.

Rasooli A, Nouri M, Khadjeh GH, Rasekh A (2004) The influences of seasonal variations on thyroid activity and some biochemical parameters of cattle. Iran, Journal of Veterinary Research 5: 1383-1391.

Ribeiro LN, Ribeiro NM, Bozzi R, Givisiez ENP, Costa GR (2014) Physiological and biochemical blood parameters of goats subjected to heat stress. Journal of Biomedical and Environmental Sciences 09: 29-35.

Richards SA (1973) Temperature Regulation. Wykeham Publications. London, Great Britain. 212p.

Rowlands GJ, Little W, Kitchenharn BA (1977) Relationships between blood composition and fertility in dairy cows - a field study. Journal of Dairy Research 44: 1-7.

SAS (2004) SAS/STAT: User's guide statistics released 9.1, 2nd Ed. SAS Institute, Inc. Cary, NC, USA. $5136 \mathrm{p}$.

Sejian V, Maurya VP, Naqvi SMK (2010a) Adaptive capability as indicated by endocrine and biochemical responses of Malpura ewes subjected to combined stresses (thermal and nutritional) in a semi-arid tropical environment. International Journal of Biometeorology 54: 653-661.

Sejian V, Maurya VP, Naqvi SMK (2010b) Adaptability and growth of Malpura ewes subjected to thermal and nutritional stress. Tropical Animal Health and Production 42: 1763-1770.

Silanikove N (2000) Effects of heat stress on the welfare of extensively managed domestic ruminants. Livestock Production Science 67: 1-18. 
Silva JAR, Araújo AA, Júnior JBL, Viana RB, Santos NFA, Garcia AR (2011) Perfil hematológico de búfalas da raça Murrah, criadas ao sol e à sombra, em clima tropical da Amazônia Oriental. Acta Amazónica 41: $425-430$.

Singh SP, Hooda OK, Kundu SS, Singh S (2012) Biochemical changes in heat exposed buffalo heifers supplemented with yeast. Tropical Animal Health and Production 44: 1383-1387.

Sivakumar AVN, Singh G, Varshney VP (2010) Antioxidant supplementation on acid base balance during heat stress in goats. Asian-Australasian Journal of Animal Science 23: 1462-1468.

Srikandakumar A, Johnson EH, Mahgoub O (2003) Effect of heat stress on respiratory rate, rectal temperature and blood chemistry in Omani and Australian Merino sheep. Small Ruminant Research 49: 193-198.

Sullivan ML, Cawdell-Smith AJ, Mader TL, Gaughan JB (2011) Effect of shade area on performance and welfare of short-fed feedlot cattle. Journal of Animal Science 89: 2911-2925.

Valtorta SE, Gallardo MR (2004) Evaporative cooling for Holstein dairy cows under grazing conditions. International Journal of Biometeorology 48: 213-217.

Wang H, Huang M, Li S, Wang S, Dong S, Cui D, et al. (2015) Hematologic, serum biochemical parameters, fatty acid and amino acid of longissimus dorsi muscle in meat quality of Tibetan sheep. Acta Scientiae Veterinariae 43: 1306.

Wojtas K, Cwynar P, Kołacz R (2014) Effect of thermal stress on physiological and blood parameters in Merino sheep. Bulletin of Veterinary Institute Pulawy 58: 283-288. 
